\title{
Special section on the 13th International Symposium on Flow Visualization
}

\author{
Kenneth D. Kihm • Jean Pierre Prenel
}

Published online: 20 March 2010

(C) Springer-Verlag 2010

The 13th International Symposium on Flow Visualization (ISFV 13, http://isfv13.univ-fcomte.fr) was held at the Acropolis Convention Center, Nice, France on July 1-4, 2008. The 12th French congress on visualization in fluid mechanics (Fluvisu 12) was jointly held at the same venue. Every 2 years, the ISFV series gathers the international communities of Fluids and Thermal Engineering around the common theme of imagery and optical measurements, including the development of new visualization techniques, visualization of transport phenomena, characterization of fields of velocity, temperature and pressure, and of course, the validation of theoretical models.

The ISFV-13 symposium attracted a total of 298 participants from 27 countries and was organized in 40 standard sessions, four invited lectures, and a poster exhibition. The standard sessions included Acoustics, Specific PIV/ PTV, Periodic Phenomena, Aeronautics and Space, High Speed, Multiphase, Turbulence/Vortices, Computer Visualization, Industrial Applications, DGV/DPV, Cavities, Refractometry, Stereo and 3D, Heat and Mass Transfer, BOS/Stereo Schlieren, Walls/Boundaries, Microflows, Combustion, Hybrid/Specific Methods, Coating Sensors, Interferometry, Terrestrial Vehicles, Rotating/Oscillating/ Swirling Flows, Advanced Image Processing, Optical and

\footnotetext{
K. D. Kihm (ه)

Mechanical, Aerospace, and Biomedical Engineering Department, University of Tennessee, Knoxville, TN 37996, USA

e-mail: kkihm@utk.edu

\section{J. P. Prenel}

Department of Energy and Multi-Physical Systems, FEMTO-ST Institute, University of Franche Comté/CNRS 6174, 2 Avenue Jean Moulin, Parc Technologique, 90000 Belfort, France
}

Electrical Manipulations, Natural Life, Human Life, Earth and Environment, and Art and Learning. In addition, more than 40 posters were displayed throughout the duration of the conference.

The special committee of eight internationally recognized researchers (Kyung Chun Kim, Sang Joon Lee, Osamu Mochizuki, Robert Nelson, Jean-Michel Desse, Daniel H. Fruman, Godfrey Mungal, and Kenneth D. $\mathrm{Kihm}$ ) attended the presentation sessions to invite selected authors to submit their manuscripts for the special section for Experiments in Fluids. A total of 20 conference proceeding papers were solicited, 15 authors responded with manuscript submissions, and seven completed their revisions as finally accepted contributions. This special section begins with a review article on the surface plasmon resonance (SPR) imaging technique as a new far-field visualization tool for near-field transport characterization. The second study presents an experimental study with the use of PIV for the vortex flow characteristics along a sharp-edged delta wing with apex strake. A third study presents the "Interfacial PIV" technique for application in near-wall velocity profile determinations. The fourth study proposes a new PTV image processing algorithm adopted from an ant colony optimization. The next study in this section deals with supersonic flows for characterization of shock wave discontinuities inside a shock tube using nanosecond space discharge. A sixth study presents planar fluorescence imaging for identification of bubbles in an axisymmetric two-phase jet flow. The final paper in this section presents the effect of rim-shroud clearance on flows around a rotating disk.

Two International Awards were presented during the plenary session to two international experts for their outstanding contributions to the field of flow visualization: (1) Dr. Juergen Kompenhans, DLR, Germany, Asanuma 
Award 2008, and (2) Prof. Kenneth. D. Kihm, University of Tennessee, Knoxville, USA, Leonardo da Vinci Award 2008.

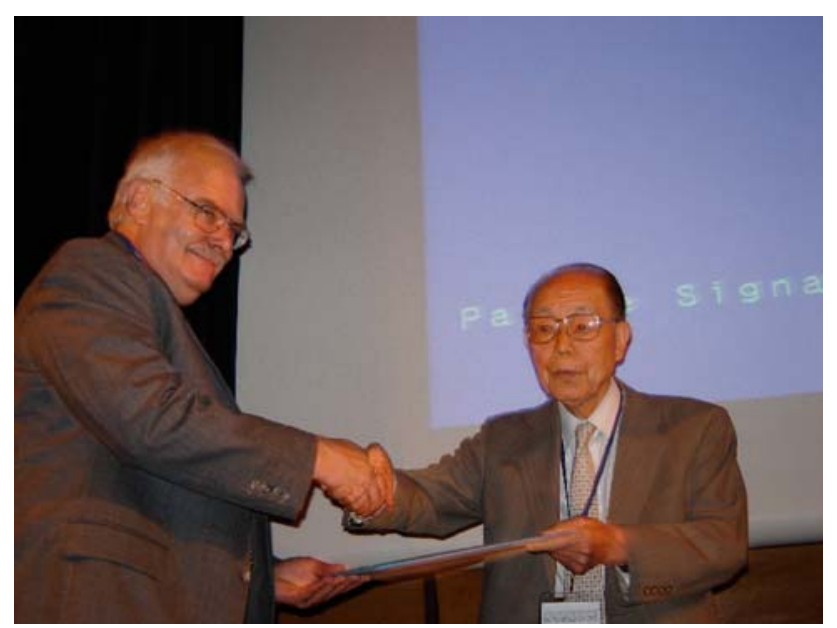

Dr. Juergen Kompenhans was awarded the 2008 Asanuma Award by Prof. Yasuki Nakayama

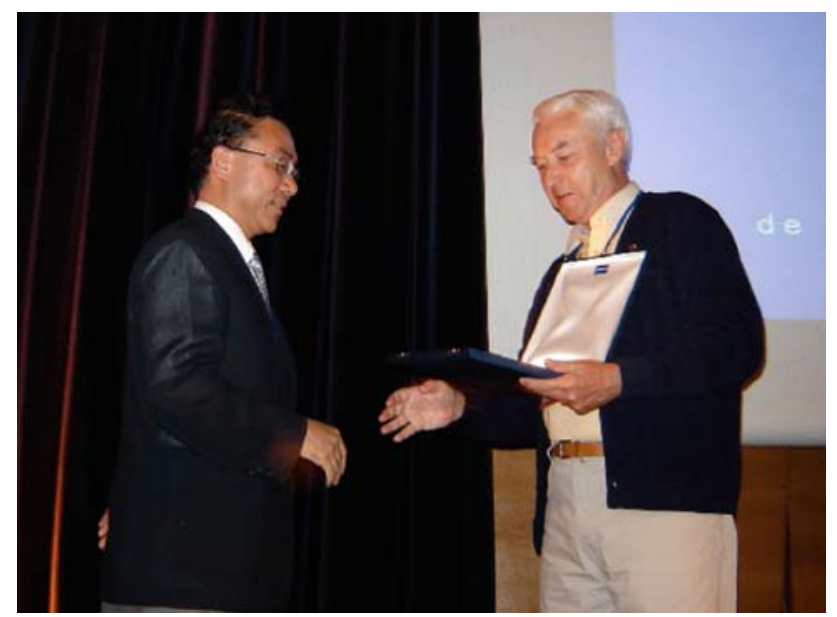

Prof. Kenneth D. Kihm was awarded the 2008 Leonardo da Vinci Award by Prof. Jean Pierre Prenel
We would like to extend our sincere thanks for all the participants, authors, exhibitors and session chairs for the success of this symposium. Special thanks are given to the French organizing team, particularly to the very efficient and devoted co-chairs (Yannick Bailly and Jean Claude Champoussin), who served as webmasters, secretaries, coordinators, and even travel agents. The financial supports from French Public Authorities and French Establishments have been greatly appreciated as well as the personal support of the FEMTO Institute Manager, Michel de Labachelerie, together with miscellaneous but invaluable help from the Research Promotion Service of the institute. Last but not least, we would like to cordially invite you to attend the 14th ISFV conference to be held on June 21-24 in 2010 in Daegu, Korea (Conference Chair: Prof. Kyung Chun Kim, http://www.isfv14.org/).

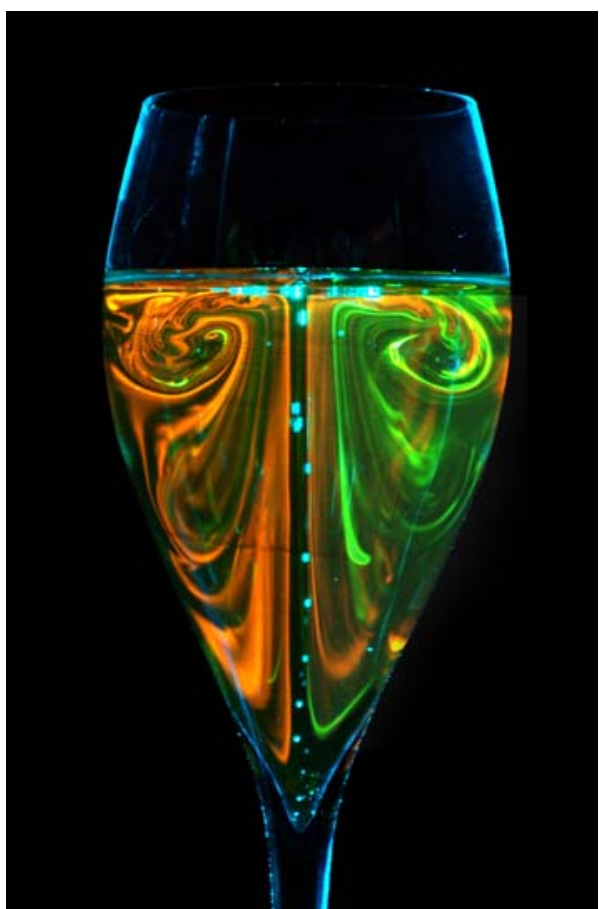

The winner of the imaging beauty by Guillaume Polidori of France 